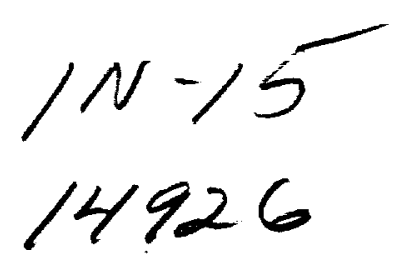

NASA Contractor Report 187138

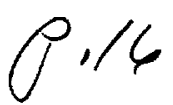

\title{
The Application of Neural Networks to the SSME Startup Transient
}

Claudia M. Meyer and William A. Maul Sverdrup Technology, Inc.

Lewis Research Center Group

Brook Park, Ohio

June 1991

Prepared for

Lewis Research Center

Under Contract NAS3-25266

\section{N/Sก}

National Aeronautics and

Space Administration 
THE APPLICATION OF NEURAL NETWORKS TO THE SSME STARTUP TRANSIENT

\author{
Claudia M. Meyer \\ and \\ William A. Maul \\ Sverdrup Technology, Inc. \\ Lewis Research Center Group \\ Brook Park, Ohio 44142
}

\begin{abstract}
$\underline{\text { Abstract }}$
Feedforward neural networks were used to model three parameters during the Space Shuttle Main Engine startup transient. The three parameters were the main combustion chamber pressure, a controlled parameter; the high pressure oxidizer turbine discharge temperature, a redlined parameter; and the high pressure fuel pump discharge pressure, a failure-indicating performance parameter. Network inputs consisted of time windows of data from engine measurements that correlated highly to the modeled parameter. A standard backpropagation algorithm was used to train the feedforward networks on two nominal firings. Each trained network was validated with four additional nominal firings. For all three parameters, the neural networks were able to accurately predict the data in the validation sets as well as the training set.
\end{abstract}

\title{
Nomenclature
}

a

CADS

DUM1

DUM2

e(t)

$f$

FPB

FPOV

HPFP

HPFT

HPFTP

HPOT

MCC

$n_{i}$

net $_{i}$

$o_{i}$

$o_{p}$

OPB

OPOV

PBP

$r$

rms

SSC

SSME

$t$

$\mathbf{t}_{\mathrm{p}}$

$\theta_{i}$

$u_{i}(t)$

$w_{i j}$

$y(t)$

activation function of hidden and output layer nodes

command and data simulator

open-/closed-loop variable for the MCC pressure model

pre-/post-prime time and open-/closed-loop variable for the

HPOT discharge temperature model

additive uncorrelated noise

nonlinear function describing relationship between system variables

fuel preburner

fuel preburner oxidizer valve

high pressure fuel pump

high pressure fuel turbine

high pressure fuel turbopump

high pressure oxidizer turbine

main combustion chamber

the window size of function input $u_{i}(t)$

the net input to node $i$

the output of node $i$

the predicted value for input pattern $p$

oxidizer preburner

oxidizer preburner oxidizer valve

preburner boost pump

number of model input variables

root mean squared

Stennis Space Center

Space Shuttle Main Engine

time

the target value for input pattern $p$

the bias term for node $i$

a function input variable

weight connecting nodes $i$ and $j$ in adjacent layers

the function output 


\section{$\underline{\text { Introduction }}$}

Multilayer feedforward neural networks were used to model critical parameters of the Space Shuttle Main Engine (SSME) during the six-second startup transient. The three parameters selected for modeling were the Main Combustion Chamber (MCC) pressure, a controlled variable; the High Pressure Oxidizer Turbine (HPOT) discharge temperature, a redlined parameter; and the High Pressure Fuel Pump (HPFP) discharge pressure, a parameter cited in failure investigation summaries for providing early failure indications.' These models were developed for eventual incorporation into a real-time advanced safety system or a post-test diagnostic system. Accurate models of parameters during the SSME startup transient are needed to improve the fault detection and isolation capability of condition monitoring systems during the first six seconds of engine operation.

The current SSME safety system employs basic redline limits and ignition confirm criteria. The redlines have upper and/or lower limits assigned to them. Limit monitoring commences at scheduled times during startup.' In addition, ignition confirm criteria are administered to three parameters during startup to ensure that ignition has occurred. Failure to meet any of the criteria results in engine shutdown. ${ }^{2}$ Current post-test analysis of the start transient consists of visually comparing parameters to two standard deviation limits and to previous start transients.

Recent SSME condition monitoring activities have focused on both real-time safety monitoring and post-test diagnostics. ${ }^{1 .+8}$ These efforts have demonstrated the potential of advanced algorithms to provide improved failure detection capability during ground test firings, thus reducing engine and/or test stand damage, and to facilitate the post-test diagnostic process. Efforts in advanced safety monitoring systems for the SSME have emphasized mainstage failure detection. ${ }^{1,4-7}$ Startup is difficult to monitor because of the highly complez and nonlinear nature of the SSME dynamics. Accurate models of parameters during startup would provide improved engine and sensor failure detection capability during the first seconds of engine operation. The models would also provide additional information for fault detection and isolation when used as part of an automated engine diagnostic system. ${ }^{8}$ In both cases, the residual between the actual and predicted values must be analyzed to detect an anomalous engine condition or to identify an engine or sensor failure.

The ability of feedforward networks to model nonlinear systems has been demonstrated in the literature; it has been shown that neural networks with only one hidden layer can uniformly approximate any continuous function. ${ }^{9-11}$ Neural networks are well-suited for problems in which the exact relationships between sensor measurements are complex or unknown." In this investigation, neural networks were used to learn the relationships among several SSME sensor measurements during the startup transient. A standard backpropagation algorithm was used to train the networks. The training set consisted of Command and Data Simulator (CADS) and facility data from the first six seconds of two nominal firings, B1070 and B1072. The trained networks were validated using data from four additional nominal firings: B1073, B1074, B1075 and B1077. The first two characters of a test firing designation indicate the test stand at Stennis Space Center (SSC) on which the firing took place, and the last three numbers indicate the test number. In addition to $B 1$, there are two other SSME test stands at SSC, A1 and A2. In order to improve model prediction accuracy, several issues related to the engine system and the neural network architecture were addressed when training the networks. These issues included the selection of measurements in the input vector, the number of nodes in the hidden layer, the transition from open loop to closed-loop control, and the prime times of the MCC, the Fuel Preburner (FPB) and the Oxidizer Preburner (OPB). Performance of the trained networks, or models, on the training and validation sets was measured by the total root mean squared (rms) error over all of the output patterns of a given set and by the maximum percent deviation between actual and predicted values. 


\section{$\underline{\text { Analysis }}$}

Theory

The mathematical relationship between observed system variables, or sensor outputs, can be very complex and the exact form is usually unknown. In practice, the modeling of real-world systems based on observed system variables is achieved by choosing a model set of known functions that is dense in the space of continuous functions. " Polynomial functions are an example of such a model set. Feedforward networks with nonlinear processing elements have been considered as an alternative model set and have been successfully used to model nonlinear dynamical systems. ${ }^{9-11}$ Because neural networks can uniformly approximate any continuous function, the neural network approach has some advantages over more conventional methods. Polynomial methods, for example, have difficulties in the presence of nonpolynomial nonlinearities while neural networks can accurately model both polynomial and nonpolynomial nonlinearities.

Consider the nonlinear function, $f$, that describes the relationship between several system variables:

$$
y(t)=f\left(u_{1}(t-1), \ldots, u_{1}\left(t-n_{1}\right), u_{2}(t-1), \ldots, u_{2}\left(t-n_{2}\right), \ldots, u_{i}(t-1), \ldots, u_{i}\left(t-n_{i}\right), \ldots, u_{r}(t-1), \ldots, u_{r}\left(t-n_{r}\right)\right)+e(t)
$$

where $y(t)$ is the function output, $u_{i}(t)$ is a function input variable, $n_{i}$ is the window size for input variable $u_{i}(t)$, and $e(t)$ is additive uncorrelated noise. ${ }^{11}$ One of the function inputs, $u_{i}(t)$, can represent autoregressive information. The total number of input variables is $r$. Neural networks are used to approximate the function $f$ based on observed system variables, $u_{i}(t)$.

Feedforward networks with a single hidden layer were used in this investigation. Each layer consists of processing elements called nodes; the structure of a feedforward network is given in Fig. 1. Each node of a given layer receives input from all of the nodes in the previous layer and sends its output to each node in the following layer. The connections are unidirectional and have weights associated with them. There are no connections between nodes within a layer and no connections bridging layers. The relationship between the input and the output of a node in the hidden or output layers is determined by the activation function, $a$. The commonly used sigmoid activation function was employed in this study: ${ }^{2}$

$$
a\left(\text { net }_{i}\right)=o_{i}=\frac{1}{1+e^{-m e_{i}}}-\frac{1}{2}
$$

where net $t_{i}$ is the net input to node $i$

$$
n e t_{i}=\theta_{i}+\Sigma w_{i} o_{j}
$$

$\theta_{i}$ is the bias term for unit $i, o_{j}$ is the output of node $j$ in the previous layer, and $w_{i j}$ is the weight between the two nodes $i$ and $j$. A bias is similar in function to a threshold and is treated as a weight connected to a node that is always on. The values of the weights and biases are adjusted during the learning process. ${ }^{13}$

The multiple-input single-output network can be described as a mapping function which uniformly approximates the function $f$ given by Eq. (1). The approximation is achieved by using the backpropagation algorithm, a supervised learning procedure based on the Generalized Delta Rule. ${ }^{13}$ Backpropagation computes the weights and bias terms to minimize the mean squared error between the values predicted by the network and the desired values. In this investigation, the weights and biases were updated after every complete pass through the training data. The learning rate and momentum determined the amount by which the weights were updated and were set to achieve rapid learning without significant oscillations in the error. ${ }^{13}$ 
Two parameters were used to assess the performance of the trained networks: the total rms error of the output layer and the maximum percent error of a training or validation set. The total rms error represents the average of the output error squared over all of the patterns in a training or validation set:

$$
\text { total rms error }=\sqrt{\frac{\sum_{p=1}^{n \text { paturns }}\left(t_{p}-o_{p}\right)^{2}}{\# \text { patterns }}}
$$

where $t_{p}$ is the target or desired output and $o_{p}$ is the network output for pattern $p$. The second performance measure is the maximum percent error of a training or validation set, where the percent error of a particular pattern, $p$, is given by:

$$
\text { percent error }=\frac{t_{p}-o_{p}}{t_{p}}
$$

\section{Application to SSME Data}

In using feedforward neural networks to model the SSME startup transient, several issues related to the network architecture were addressed. These included the selection of the measurements (including autoregressive information) in the input vector, the window size, and the number of nodes in the hidden layer. In addition, several engine system conditions required monitoring in order to improve network prediction accuracy. These were sensor failures, the initiation of closed-loop control during the start sequence, and the realization of full faceplate burning, or prime time, of the three combustion chambers (MCC, FPB, and OPB). These issues are discussed in this section.

The engine measurements in the model input vector were determined by computing the correlation coefficient between the parameter to be modeled and the remainder of the measurements in the CADS and facility data sets. Many parameters were found to be highly correlated due to their strong response to changes in power level. Physically relevant, non-redundant measurements with high correlation coefficients were chosen; these parameters are given in Table 1. It should be noted that sensor failures affected the choice of measurements. The HPFP discharge temperature, for example, could not be included in the input vector for the HPFP discharge pressure, since the temperature measurement was faulty in several of the test firings used. Data from the first six seconds of test firings $\mathrm{B} 1070$ and $\mathrm{B} 1072$ were used to construct the training sets. Prior to training, all input-output pairs were normalized to fall within the range $[-0.5,0.5]$ as dictated by the activation function given by Eq. (2). The normalization was achieved by selecting maximum values for the input and output variables based on the data in all of the training and validation sets.

The impact of including autoregressive information in the network input was investigated for all three parameters. Autoregressive information did not improve the predictive accuracy of the MCC pressure and the HPFP discharge pressure models and was not used in the final models for these parameters. The HPOT discharge temperature model, on the other hand, exhibited a large improvement in prediction accuracy when autoregressive information was added; the total rms error decreased by a factor of 1.8 . Autoregressive data increased the size of the input layer and hence was accompanied by increases in training and processing times. Autoregressive information was retained in the final HPOT discharge temperature model, however, because significant improvements in the quality of the predicted signal were realized.

The window size was chosen based on behavior observed for the MCC pressure. For a given neural network model, the same window size was used for each input variable, $u_{i}(t)$. Therefore, the 
number of nodes in the input layer was equal to the number of input variables, $r$, multiplied by the window size. A window size of one corresponds to one sampling interval, or $40 \mathrm{msec}$. Window sizes of ten, five, and one were examined. The total rms error for the MCC pressure increased by a factor of 1.3 when the window size was changed from ten to five, and increased by a factor of 2.15 when the window size was changed from five to one. A window size of ten was chosen in this investigation because of the improved quality of the predicted signals. Window sizes larger than ten were not considered for two reasons. First, larger window sizes increase the time until the first predicted value is available to the safety or diagnostic system thereby reducing the period of coverage during the startup transient. Second, larger window sizes increase the number of nodes in the input layer, thereby increasing the processing requirements.

The size of the hidden layer was chosen by varying the number of hidden nodes used to train the MCC pressure networks. When the number of hidden nodes was increased from 10 to 20 , no improvement in the total $\mathrm{rms}$ error was observed. In addition, the larger hidden layer increased the number of weights and hence the training time. Training times are important since it is anticipated that the networks will require periodic updating as new test data becomes available. Furthermore, test-stand specific networks may be required. Thus, three networks would be needed for each parameter. Excessive training times would prevent practical implementation of these models. Larger layers also increase the processing time when the network is being used in a failure detection or diagnostic mode. Ten hidden nodes were used for all subsequent simulations.

An important system condition which was taken into account during network training was the closed-loop thrust and mixture ratio control of the SSME. Engine thrust is directly proportional to the MCC pressure. Complete closed-loop control is achieved at engine start +3.6 seconds. The error between actual and desired main combustion chamber pressures drives the oxidizer preburner oxidizer valve (OPOV) and the proper mixture ratio is maintained by adjusting the Fuel Preburner Oxidizer Valve (FPOV). All other valves are scheduled. ${ }^{14}$ Closed-loop control was taken into account when modeling the MCC pressure and the HPOT discharge temperature, since the input vectors for these two parameters included the FPOV and OPOV. For the MCC pressure model, a dummy variable, DUM1, was added to each time slice in the input layer to indicate whether the data at that time slice represented partially open or completely closed-loop behavior:

$$
\text { DUMI }=\left\{\begin{array}{l}
-0.25,0<t<3.6 \\
+0.25, t>3.6
\end{array}\right.
$$

A slightly modified version of Eq. (6) was used for the HPOT discharge temperature and is given later in this section.

Each of the modeled parameters is closely associated with one of the combustors and is therefore strongly affected by the prime time of the relevant chamber. The MCC prime time is defined as the time when the MCC pressure reaches 100 psia. The OPB prime time is determined by monitoring the HPOT discharge temperature, and occurs when the temperature changes from a decreasing to an increasing trend. Although the HPFP discharge pressure is not used in determining the FPB prime time, it is strongly affected by the prime time of this chamber. The FPB prime time is defined as the point at which the High Pressure Fuel Turbopump (HPFTP) shaft speed exhibits a sharp increase in slope. During a nominal firing, the FPB primes first, followed by the MCC and the OPB. ${ }^{16}$ The prime times for the test firings used in this investigation were obtained using the above criteria. The dependence of model use on the determination of prime times is not unreasonable, even for a real-time safety system, since complicated calculations are not required. Typical prime times for the FPB, MCC and OPB are $1.37,1.44$, and 1.62 seconds from engine start, respectively. ${ }^{16}$

The effect of prime time on model prediction accuracy was investigated for all three parameters. The solid curves in Figs. 2(a), 2(b), and 2(c) give mean values for the MCC pressure, the HPFP discharge pressure and the HPOT discharge temperature, respectively, during the first six 
seconds of engine operation. The mean values are currently used in the post-test SSME data reviews and represent the average behavior of the parameters over a large number firings considered nominal by engine experts. ${ }^{17}$ Sharp increases in the three parameters correspond approximately to the prime times listed above. The MCC pressure network was first trained using the entire six second startup transient. Errors approaching 100 percent of the actual values were observed prior to prime of the MCC. Figure 3 shows the percent error (Eq. (5)) as a function of time for a test firing in the training set, B1070. These large errors are due to the near zero values of the MCC pressure prior to the MCC prime time. The MCC prime for this test, indicated by the vertical line, occurred at approximately $1.48 \mathrm{sec}$, and was determined by the criterion described above. When trained on only post-prime time data, the maximum percent error on a test firing in the training set was less than one percent. Like the MCC pressure, the HPFP discharge pressure is close to zero during the first second of engine operation, and large percent errors were observed between the actual and predicted values when preFPB prime time data was included in the training set. The FPB primes earlier than the MCC; therefore, the time interval during which a predicted value is not available is smaller for the HPFP discharge pressure than for the MCC pressure. Since errors approaching 100 percent of the actual value are not likely to provide useful information to a safety or diagnostic system, pre-prime time data was not included in the final models for the pressures.

The HPOT discharge temperature was trained on pre- and post-prime time data since it is nonzero prior to the prime of the OPB. This was desirable since the OPB primes the latest of all three chambers, typically around engine start +1.62 seconds. Training on post-prime time data only would significantly decrease the failure coverage for this parameter. The effect of prime time on the HPOT discharge temperature was assessed by training networks with and without a variable to indicate the transition from pre-prime to post-prime time behavior. The dummy variable indicating open-/closedloop behavior given by Eq. (6) was modified for the HPOT discharge temperature to include the transition to full faceplate burning of the OPB:

$$
D U M 2=\left\{\begin{array}{l}
0.0,0<t<O P B \text { prime time } \\
-.25, \text { OPB prime time }<t<3.6 \\
+.25, t>3.6
\end{array}\right.
$$

The introduction of the prime time variable was accompanied by a decrease in the total rms error.

\section{$\underline{\text { Results and Discussion }}$}

Data from two nominal SSME firings were used to train feedforward networks to model three parameters during portions of the SSME startup transient. Several issues were presented in the application of the feedforward networks to the MCC pressure, the HPFP discharge pressure and the HPOT discharge temperature. The network which yielded the best model for each parameter when trained on data from test firings B1070 and B1072 was subsequently tested using data from four additional nominal firings: $\mathrm{B} 1073, \mathrm{~B} 1074, \mathrm{~B} 1075$ and $\mathrm{B} 1077$. The results are discussed below.

The performance of the best model for each parameter is given in Tables 2, 3 and 4 . The total output rms error and maximum percent error, described by Eqs. (4) and (5), are given for each training and validation set. All three models used a window size of ten and a hidden layer with ten nodes. Table 1 summarizes the complete set of variables which comprised the input for each model. All models were trained for 20000 cycles. In a real-time safety or post-test diagnostic application, the number of training cycles should be chosen to achieve an optimum balance between model performance and practical implementation concerns. The MCC pressure model used to generate the values in Table 2 was trained on post-prime time data only and included the open-/closed-loop variable given by Eq. (6). The HPFP model evaluated in Table 3 was trained on post-FPB prime time data. The HPOT discharge temperature model of Table 4 was trained on the entire first six seconds of engine operation. It included autoregressive information and the prime time and open-closed-loop variable given by Eq. (7). In all cases, the total rms error and the maximum percent error were lower on the training test firings than on the validation test firings. The maximum percent error on a validation set, however, 
was 2.92 percent for the MCC pressure, 4.94 percent for the HPFP discharge pressure, and 3.31 percent for the HPOT discharge temperature. These small errors indicate that networks have successfully generalized the data in the two training test firings.

The maximum percent errors can be compared to the nominal test-to test variations exhibited by the three parameters. In addition to giving the mean values, Figs. 2(a), 2(b), and 2(c) show the mean plus and minus two standard deviation values during startup for the MCC pressure, the HPFP discharge pressure, and the HPOT discharge temperature, respectively. The two standard deviation envelopes, like the mean values, are currently used in the SSME post-test data reviews. They were computed from a large number of test firings which engine experts considered nominal. ${ }^{17}$ The MCC pressure, a controlled variable, experiences the smallest test-to-test variation, while the HPOT discharge temperature experiences the largest. In all cases, the predictions of the networks were closer than the plus and minus two standard deviation variations shown in Fig. 2.

When considering the test-to-test variations shown in Fig. 2(c), it can be seen that the performance of the HPOT discharge temperature model represented the most dramatic improvement over the two standard deviation limits. The quality of the predicted signals is displayed graphically for the HPOT discharge temperature in Figs. 4 and 5. Figure 4(a) gives the actual and predicted values for a test firing in the training set, B1070. The first predicted value is at .40 seconds due to the window size of ten. Figure 4(b) gives the difference between the two curves; the maximum error is less than $6 \mathrm{deg} R$. Figure 5 illustrates the performance of the same model on a validation set test firing, B1077. Actual and predicted values are shown in Fig. 5(a) and the difference between the actual and predicted values is given in Fig. $5(\mathrm{~b})$. The maximum error on this firing is less than 16 deg R. A comparison of both Fig. 4(a) and Fig. 5(a) to Fig. 2(c) indicates that the neural network is a good predictor of the HPOT discharge temperature even in areas where the temperature varies widely.

A final note is in order regarding the models generated in this investigation. Each parameter had an input vector which contained a large number of highly correlated, non-redundant engine measurements. Alternate measurements were not considered. Smaller input vectors would reduce training time, yet may also degrade model accuracy. The effect of reducing the input vector size on prediction accuracy may suggest that smaller input vectors are adequate, particularly for a real-time implementation. In addition, fault isolation becomes difficult when a given parameter appears in the input vector for a large number of parameters. The inclusion of autoregressive information in the model of a parameter may desensitize the model to slow drifts in that parameter and therefore impede the detection of soft sensor failures or engine degradations which are manifested as drifts. The ability of feedforward networks to accurately model critical SSME parameters during the startup transient has been demonstrated; the needs and limitations of the safety or post-test diagnostic system will determine many of the issues involved in model construction.

\section{Concluding Remarks}

Feedforward neural networks were used to accurately model three critical parameters during portions of the SSME startup transient. The formulation of accurate models is the first step towards improved startup fault detection and isolation. Once the models have been established, the residuals between the actual and synthesized values must be analyzed for indications of engine or sensor anomalies. Furthermore, fault isolation logic must be applied to the residuals in order to identify the source of the failure; more detailed fault isolation will be possible in a post-test diagnostic system. The models, along with the appropriate fault detection and isolation logic, can then be incorporated into an existing safety or diagnostic system. As part of an advanced safety system, for example, the neural network models could assist the ignition confirm criteria in ensuring safe engine operation during startup.

In this investigation, the MCC pressure, the HPFP discharge pressure, and the HPOT discharge temperature were simulated using time windows of data from highly correlated engine 
measurements. Two nominal firings were used to train the networks and four additional nominal firings were used to validate the trained networks. All six firings were from the same test stand. In all cases, the errors were smaller on the training sets than on the validation sets. The maximum percent errors on the validation sets were, however, all less than 5 percent. The small errors indicate that the networks have successfully generalized the information in the training sets. Furthermore, the errors fell within the two standard deviation envelopes typically observed for the three parameters during startup. These envelopes are currently used to do a post-test assessment of the parameters. The HPOT discharge temperature model represented the most dramatic improvement over the plus and minus two standard deviation limits because the HPOT discharge temperature exhibits larger test-to-test variations than the MCC pressure and the HPFP discharge pressure.

Model prediction accuracy was achieved by constructing the training sets to reflect important system conditions and by adjusting variables related to the network architecture. Dramatic improvements in network performance were realized when the pressures were trained only on data following the prime time of the relevant chamber. Although training on only post-prime time data reduces the failure coverage for the MCC pressure and the HPFP discharge pressure, the large percent errors experienced prior to MCC or FPB prime would be of little benefit to a safety or diagnostic system. The MCC and the FPB prime at approximately 1.44 and 1.37 seconds after engine start, respectively. The HPOT discharge temperature model was trained on the entire first six seconds of SSME operation; model improvement was achieved by introducing a flag to indicate the occurrence of OPB prime. The HPOT discharge temperature model was further improved by adding autoregressive information; autoregressive data did not improve the quality of the predictions for the two pressures. Larger window sizes were also found to improve model performance. A larger window size, however, reduces the failure coverage for the modeled parameter by increasing the time until the first predicted value is available to the safety or diagnostic system.

The tradeoff between model accuracy and the requirements and limitations of a safety or posttest diagnostic system was evident throughout this investigation. Improvements in model accuracy were often accompanied by longer training times and increased processing and memory requirements. This is because larger window sizes and autoregressive information increase the number of weights thereby increasing the number of computations per pass when the networks are being trained and when they are being used to model a new test firing. Although the highly parallel architecture of feedforward neural networks facilitates real-time implementation, the models are anticipated to be one component of an advanced safety system and will have limited computing and memory resources. Furthermore, some computations will be required to interpret the errors between the actual and predicted signals. Training times are important since it is anticipated that the networks will require periodic updating as new test data becomes available. All of the models constructed for this investigation were trained and validated using test data from one test stand; test stand specific models may be required. Therefore, a large number of models may require maintenance. All networks were trained for 20000 cycles since model accuracy was the objective of this investigation. Practical considerations may suggest a smaller number of training cycles.

\section{Acknowledgment}

This work was supported by the NASA Lewis Research Center under contract NAS3-25266 with Larry P. Cooper as monitor.

\section{References}

1. Taniguchi, M.H. Failure Control Techniques for the SSME, Phase I Final Report. NASA CR$179224,1986$.

2. Roth, P. Computer Program Contract End Item, Flight 4C Configuration, Space Shuttle Main Engine Controller Operational Program, Part 1. CP406R0001, Revision F, Rockwell 
International/Rocketdyne Division, November 1988.

3. Vaughan, D. Private Communication - Data Summary Package for Test Firing B1063: Start Two Sigma. Martin Marietta Corporation at Marshall Space Flight Center, January 1990.

4. Hawman, M.H., Galinaitis, W.S., Tulpule, S., and Mattedi, A. Framework for a Space Shuttle Main Engine Health Monitoring System, Final Report. NASA CR-185224, May 1990.

5. Nemeth, E. Health Managenent System for Rocket Engines, Final Report. NASA CR-185223, June 1990.

6. Meyer, C.M. and Zakrajsek, J.F. Rocket Engine Failure Detection Using System Identification Techniques. AIAA Paper 90-1993, July 1990.

7. Maul, W.A. Multi-sensor Analysis Techniques for SSME Safety Monitoring. AIAA Paper 901990, July 1990.

8. Zakrajsek, J.F. The Development of a Post-Test Diagnostic System for Rocket Engines. AJAA Paper 91-2528, June 1991.

9. Lapedes, A. and Farber, R. Nonlinear Signal Processing Using Neural Networks: Prediction and System Modelling. Los Alamos National Laboratory Technical Report LA-UR-87-2662, July 1987.

10. Weigand, A.S., Huberman, B.A., and Rumelhart, D.E. Predicting the Future: A Connectionist Approach. Stanford PDP-90-01, April 1990.

11. Chen, S., Billings, S.A., and Grant, P.M. Non-linear Systems Identification Using Neural Networks. University of Edinburgh Research Report 370, August 1989.

12. ANSim User's Manual, Version 2.30. Science Applications International Corporation, April 1989.

13. Rumelhart, D.E. and McCleliand, J.L. Parallel Distributed Processing: Explorations in the Microstructure of Cognition, I. Cambridge, MA: MT Press, 1987.

14. SSME Orientation (Part A-Engine). Course No. ME-110(A)RIR, Rockwell International/Rocketdyne Division, August 1988.

15. Connell, S.C. Space Shuttle Main Engine Component Level Hot-fire Testing Procedure (Green Run Specification). RL00461, Revision L, Rockwell International/Rocketdyne Division, January 1991.

16. Hooper, Taylor. Private Communication. Martin Marietta at NASA Marshall Space Flight Center, December 1990.

17. Foust, D. Private Comnunication - SSME Startup Two Sigma Database. Martin Marietta Corporation at Marshall Space Flight Center, September 1990. 


\begin{tabular}{|c|c|c|c|}
\hline $\mathrm{u}_{\mathrm{i}(\mathrm{t})} \mathrm{y(t)}$ & $\begin{array}{c}\text { MCC } \\
\text { Pressure }\end{array}$ & $\begin{array}{c}\text { HPFP } \\
\text { Discharge } \\
\text { Pressure } \\
\end{array}$ & $\begin{array}{c}\text { HPOT } \\
\text { Discharge } \\
\text { Temperature } \\
\end{array}$ \\
\hline FPOV & $\mathbf{x}$ & & $\mathbf{x}$ \\
\hline OPOV & $\mathbf{x}$ & & $\mathbf{x}$ \\
\hline HPFP Discharge Pressure & $\mathbf{x}$ & & \\
\hline HPFP Coolant Liner Pressure & & $\mathbf{x}$ & \\
\hline FPB Chamber Pressure & $\mathbf{x}$ & $\mathbf{x}$ & \\
\hline PBP Discharge Pressure & $\mathbf{x}$ & & $\mathbf{x}$ \\
\hline HPFT Discharge Temperature & $\mathbf{x}$ & $\mathbf{x}$ & $\mathbf{x}$ \\
\hline HPOT Discharge Temperature & $\mathbf{x}$ & & $x^{1}$ \\
\hline OPB Chamber Pressure & $\mathbf{x}$ & & $\mathbf{x}$ \\
\hline HPFP Shaft Speed & & $\mathbf{x}$ & \\
\hline Engine Fuel Flowrate & $\mathbf{x}$ & $\mathrm{x}$ & $\mathbf{x}$ \\
\hline Engine Oxidizer Flowrate & $\mathrm{x}$ & $\mathbf{x}$ & $\mathbf{x}$ \\
\hline DUM1 & $\mathbf{x}$ & & \\
\hline DUM2 & & & $\mathbf{x}$ \\
\hline
\end{tabular}

1. autoregressive input

Table 1. Variables that appear in the neural network input for each of the three modeled parameters.

\begin{tabular}{|c|c|c|c||}
\hline $\begin{array}{c}\text { TEST } \\
\text { FIRING }\end{array}$ & T or V & $\begin{array}{c}\text { TOTAL RMS } \\
\text { ERROR }\end{array}$ & $\begin{array}{c}\text { MAX PCT } \\
\text { ERROR }\end{array}$ \\
\hline \hline B1070 & $\mathrm{T}$ & .00153 & .763 \\
\hline $\mathrm{B} 1072$ & $\mathrm{~T}$ & .00129 & .592 \\
\hline $\mathrm{B} 1073$ & $\mathrm{~V}$ & .00238 & 1.48 \\
\hline $\mathrm{B} 1074$ & $\mathrm{~V}$ & .00252 & 1.54 \\
\hline $\mathrm{B} 1075$ & $\mathrm{~V}$ & .00531 & 2.92 \\
\hline $\mathrm{B} 1077$ & $\mathrm{~V}$ & .00506 & 1.97 \\
\hline
\end{tabular}

Table 2. The performance of the MCC pressure model on Training $(T)$ and Validation $(V)$ test firings. The network was trained using post-MCC prime time data only and using the open/closed loop flag. 


\begin{tabular}{|c|c|c|c|}
\hline $\begin{array}{c}\text { TEST } \\
\text { FIRLNG }\end{array}$ & T or V & $\begin{array}{c}\text { TOTAL RMS } \\
\text { ERROR }\end{array}$ & $\begin{array}{c}\text { MAX PCT } \\
\text { ERROR }\end{array}$ \\
\hline B1070 & $T$ & .00202 & 1.59 \\
\hline B1072 & $T$ & .00158 & 1.35 \\
\hline B1073 & $V$ & .00441 & 2.03 \\
\hline B1074 & $V$ & .00401 & 1.76 \\
\hline B1075 & $V$ & .00349 & 2.33 \\
\hline B1077 & $V$ & .00670 & 4.94 \\
\hline
\end{tabular}

Table 3. The performance of the HPFP discharge pressure model on Training $(T)$ and Validation $(V)$ test firings. The network was trained using post-FPB prime time data only.

\begin{tabular}{|c|c|c|c|}
\hline $\begin{array}{c}\text { TEST } \\
\text { FIRING }\end{array}$ & T or V & $\begin{array}{c}\text { TOTAL RMS } \\
\text { ERROR }\end{array}$ & $\begin{array}{c}\text { MAX PCT } \\
\text { ERROR }\end{array}$ \\
\hline B1070 & $\mathrm{T}$ & .00125 & .960 \\
\hline $\mathrm{B} 1072$ & $\mathrm{~T}$ & .00127 & .977 \\
\hline $\mathrm{B} 1073$ & $\mathrm{~V}$ & .00381 & 3.29 \\
\hline $\mathrm{B} 1074$ & $\mathrm{~V}$ & .00358 & 2.07 \\
\hline $\mathrm{B} 1075$ & $\mathrm{~V}$ & .00787 & 3.31 \\
\hline $\mathrm{B} 1077$ & $\mathrm{~V}$ & .00495 & 2.49 \\
\hline
\end{tabular}

Table 4. The performance of the HPOT discharge temperature model on Training (T) and Validation $(V)$ test firings. The network was trained using the first sir seconds of startup and using the variable indicating transition from pre-OPB prime to post-OPB prime to closed-loop behavior. Autoregressive information was also included.

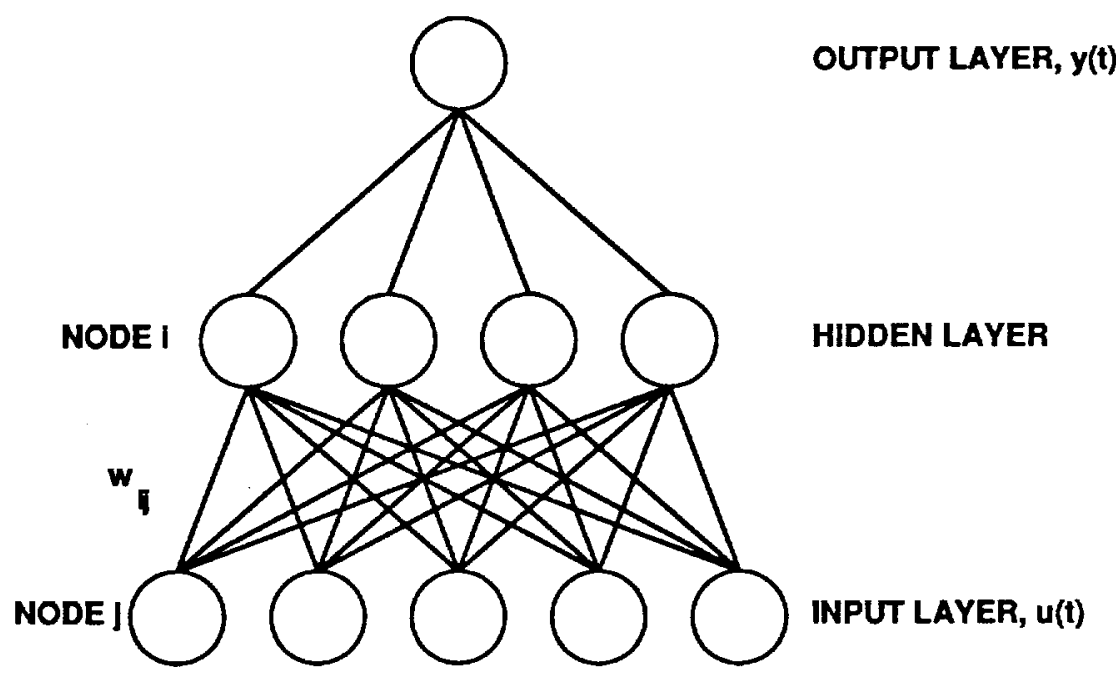

Figure 1. An example of a fully connected feedforward neural network with one hidden layer. 

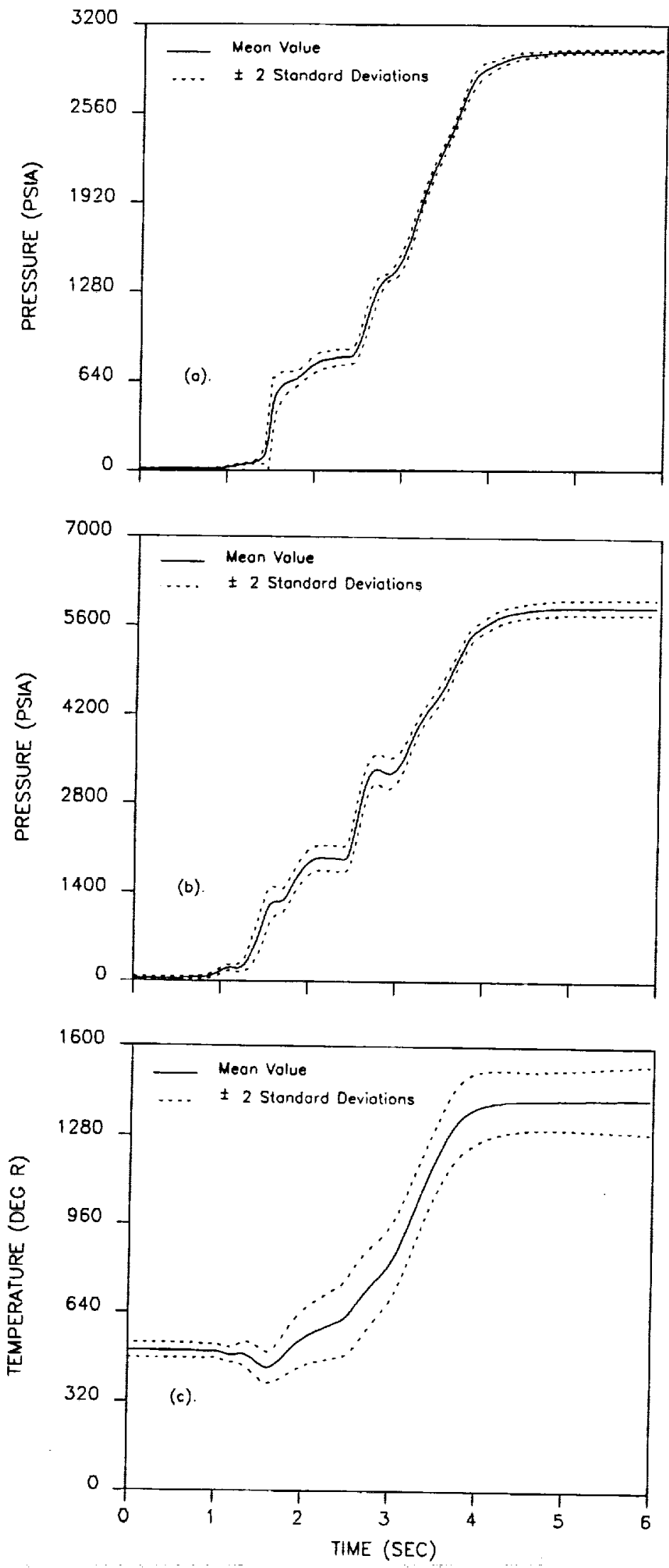

Figure 2. Nominal mean and mean plus and minus two standard deviation values during the startup transient for (a.) the MCC pressure, (b.) the HPFP discharge pressure, and (c.) the HPOT discharge temperature. 


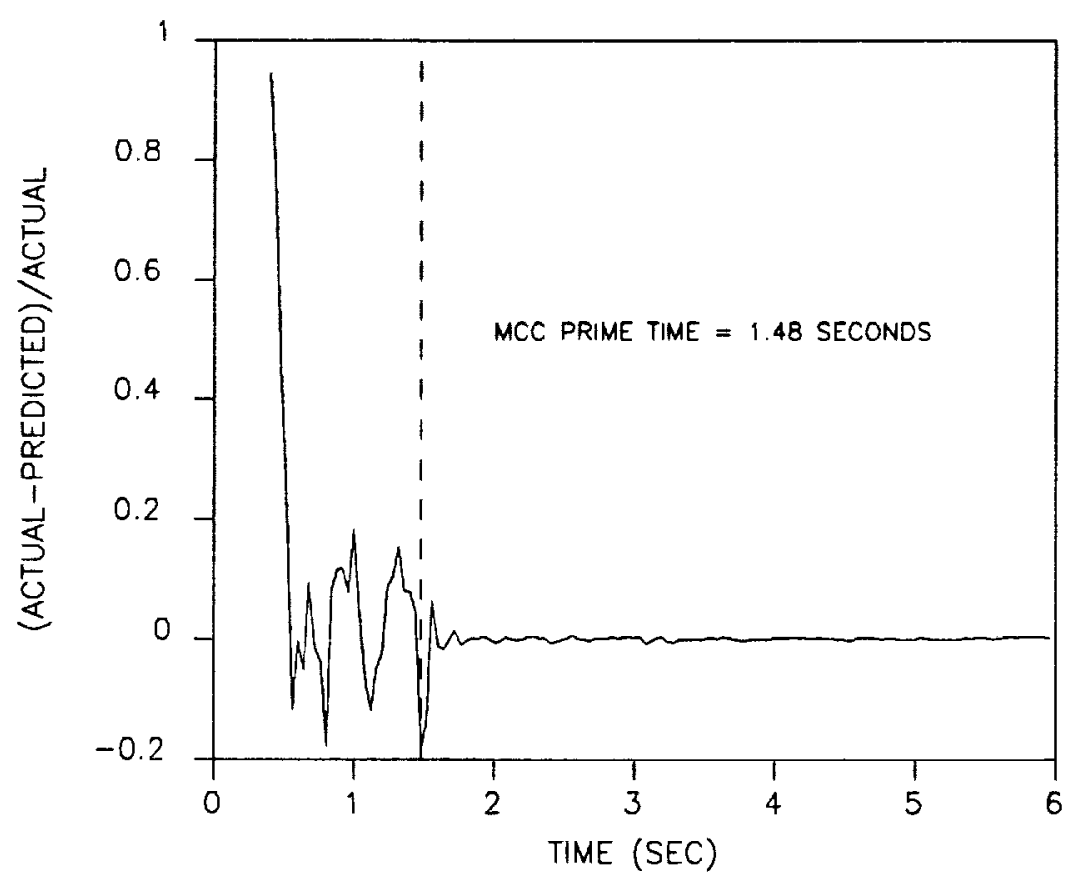

Figure 3. Percent error as a function of time for test firing B1070. The dotted vertical line indicates the MCC prime time for this test.
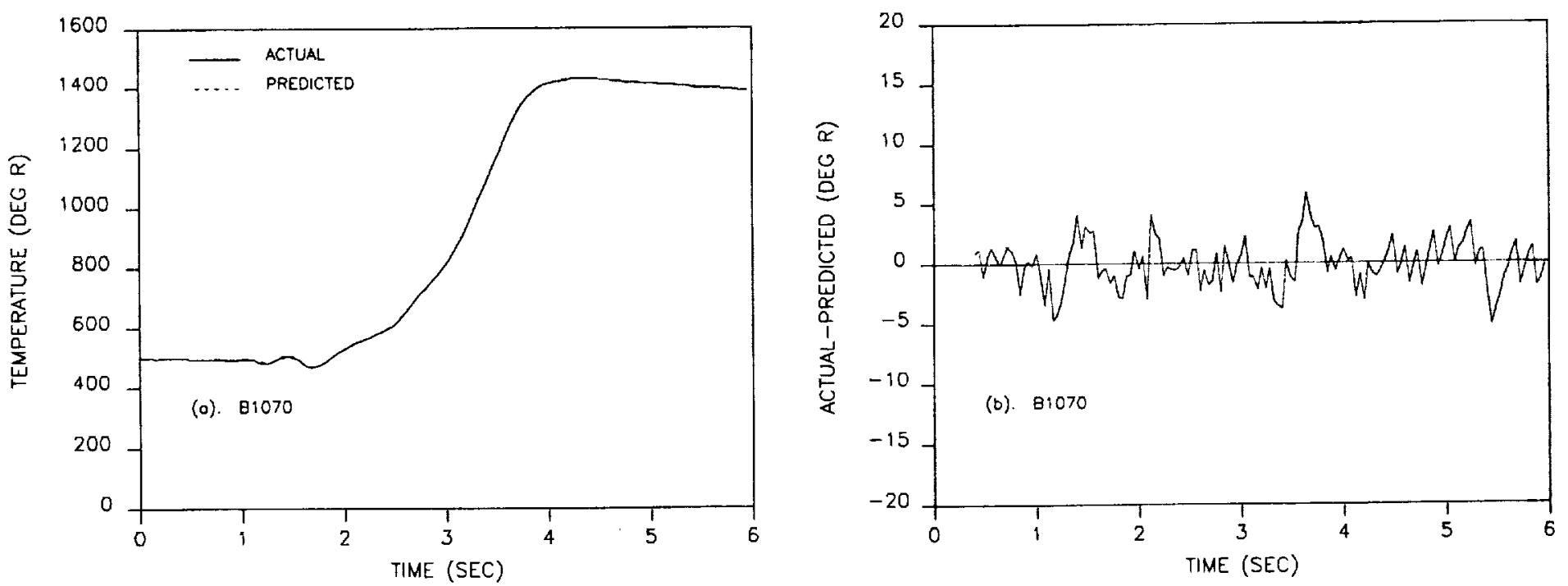

Figure 4. The performance of the HPOT discharge temperature network on a test firing in the training set, B1070: (a.) the actual and predicted signals as a function of time and (b.) the difference between the actual and predicted values. 

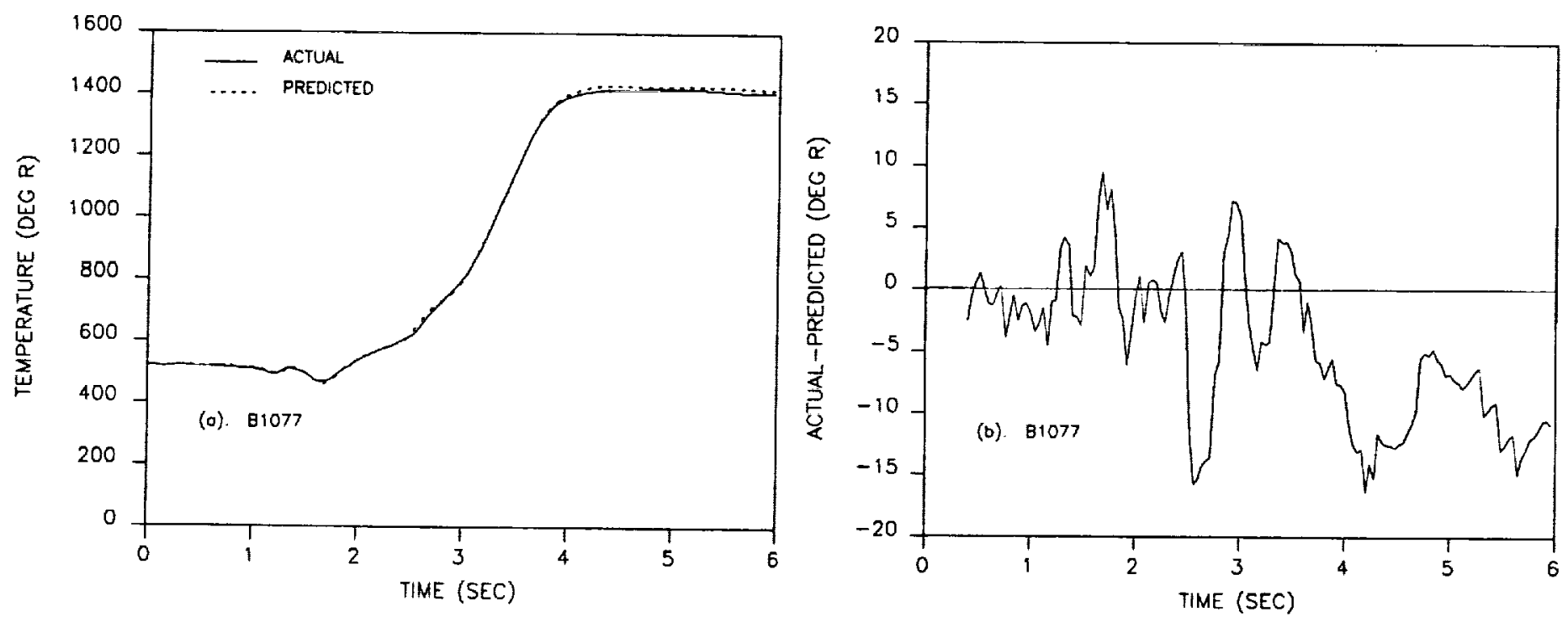

Figure 5. The performance of the HPOT discharge temperature network on a validation set test firing, B1077: (a.) the actual and predicted signals as a function of time, and (b.) the difference between the actual and predicted signals. 


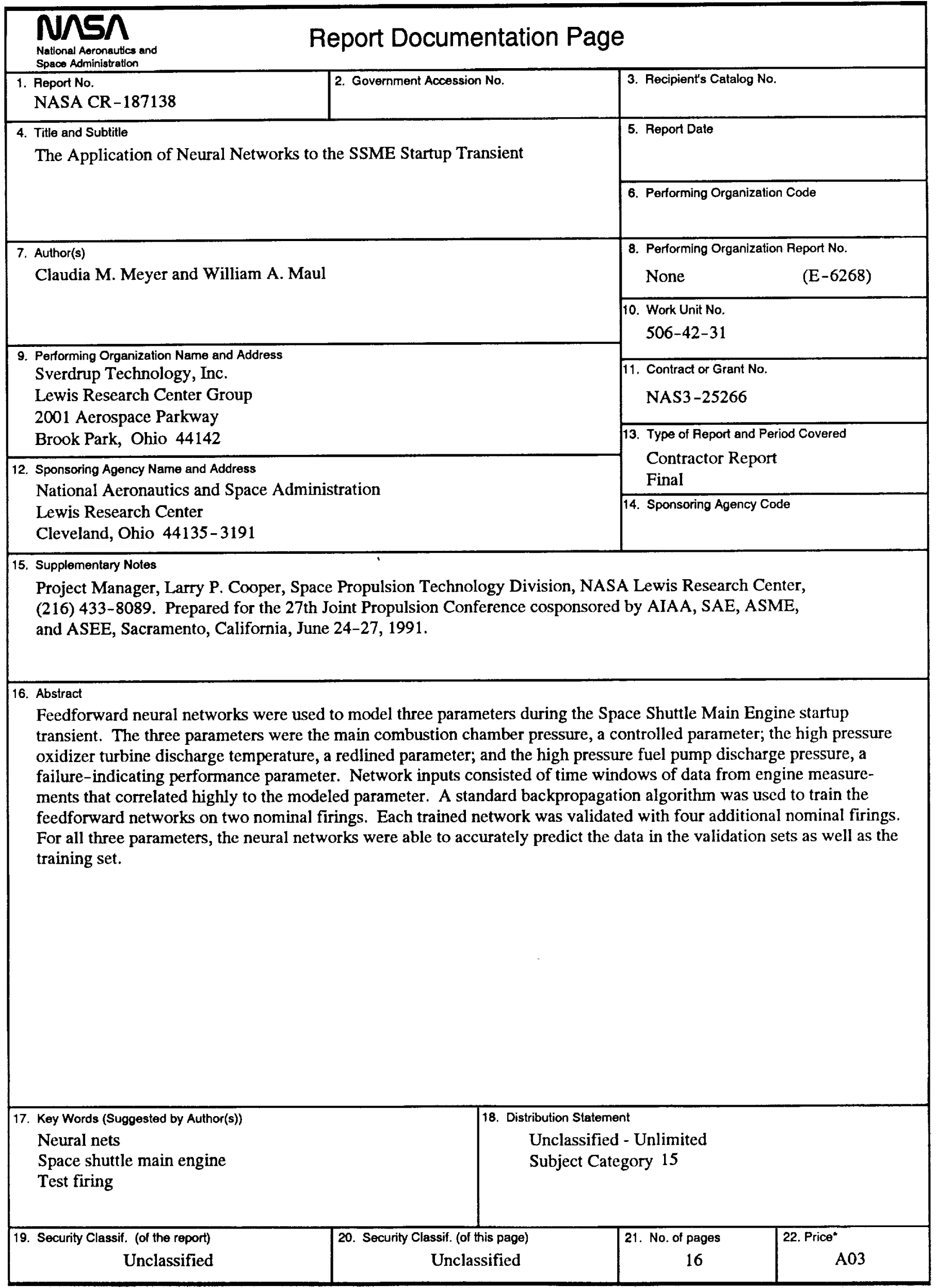


